\title{
OPTIMIZATION TECHNIQUE FOR PSEUDORANGE MULTIPATH MITIGATION USING DIFFERENT SIGNAL SELECTION METHODS
}

\author{
Valanon Uaratanawong, Chalermchon Satirapod \\ Department of Survey Engineering, Faculty of Engineering, \\ Chulalongkorn University, Bangkok, Thailand \\ e-mails: valans.wongs@gmail.com, chalermchon.s@chula.ac.th (corresponding \\ author) \\ Toshiaki Tsujii \\ Department of Aerospace Engineering, Osaka Prefecture University, \\ Osaka, Japan \\ e-mail:tsujii@aero.osakafu-u.ac.jp
}

\begin{abstract}
Nowadays, the use of multi-Global Navigation Satellite System (GNSS) has improved positioning accuracy in autonomous driving, navigation and tracking systems utilized by general users. However, signal quality in urban areas is degraded by poor satellite geometry and severe multipath errors, which may disturb up to a hundred-meter-ranging error as a consequence. In this study, the performance of several satellite selection methods in multipath mitigation was evaluated, based on the concept that better quality signals and more accurate solutions will be obtained, the more multipath signals can be excluded. Three methods were performed and compared: 1) azimuth-dependent elevation mask based on fisheye image technique, 2) receiver autonomous integrity monitoring (RAIM), and 3) signalto-noise ratio (SNR) mask in the SPP method. To examine the effect of the satellite selection methods on multipath error, the static test (single-point positioning (SPP) in real-time $1 \mathrm{~Hz}$ test) was performed in a multipath environment. The preliminary results showed a possible impact on improving the horizontal positioning accuracy of SPP. Among the three techniques assessed in this study, the results indicated that the SNR mask set at $36 \mathrm{~dB}-\mathrm{Hz}$ in every elevation showed the most promising result. The SNR mask method could improve positioning accuracy by up to $46.80 \%$ compared to the SPP method.
\end{abstract}

Keywords: Pseudorange Multipath, Satellite Selection Method, SPP

\section{INTRODUCTION}

In an urban environment where tall buildings and other structures can obscure the view of the sky, satellite visibility is also decreased, which negatively affects satellite geometry. As a result, the probability of non-line-of-sight (NLOS) satellite reception is increased and multipath is caused by signal reflection. These phenomena affect the positioning accuracy and availability in many positioning applications such as automotive navigation and location tracking. It can also produce large ranging errors as high as 100 meters in the most severe multipath conditions and provide wrong fixed position solutions, even if survey-grade receivers are used. For static method of positioning, it received more impact on multipath than 
kinematic positioning. Due to the lack of change in environment and signal received from the same group of visible satellites, multipath is easily received continuously. In addition, long time periods of receiving a signal means multipath occurs repeatedly on sidereal days.

From a previous study, there are many mitigation techniques used to reduce the pseudorange multipath error. These techniques can be divided into three categories: antenna design (Groves et al., 2013), receiver-based correlator design (Matera el al., 2019; Sánchez et al., 2017), and post-processing technique (Hsu et al., 2016; Iwase et al., 2013; Lin et al., 2018; Matera et al., 2019; Sánchez et al., 2017; Špánik and Hefty, 2017; Suzuki, 2011; Suzuki and Kubo, 2015; Satirapod and Rizos, 2005; Dammalage et al., 2010). Without involving modification of the antenna used or the complexity of implementing the receiver's architecture, the post-processing technique is the optimum choice for work that does not require a high-precision solution. In addition, it deals directly with multipath contaminated measurements without using external data. At present, the satellite selection method is one of the post-processing techniques widely used in the era of multi-GNSS with many more visible satellites (Suzuki, 2011; Tokura and Kubo, 2014; Tokura and Kubo, 2017). This method implementation combines NLOS and multipath detection with a mitigation technique. For detection, a sky-pointing camera with a fisheye lens can be used to display an entire field of view above an antenna in real-time. The blocked and unblocked satellites can be determined from the image. For mitigation, satellite selection is based on the concept that, if a subset of high-quality satellites is selected and low-quality satellites that are affected by multipath are excluded or down weighted, more accurate solutions are provided. To determine whether to select or remove, a measurement's quality is indicated by pseudorange residual, satellite elevation angle and signal strength, which are often used as filters to enhance positioning performance.

This study aimed to reduce the multipath errors in static positioning and evaluate the satellite selection methods for multi-GNSS in urban environments based on the assumption that NLOS satellite signals mainly cause the multipath errors received. This study performed three techniques for the satellite selection method: 1) Elevation mask based on a fisheye image, 2) RAIM with fault detection and exclusion (FDE) function, 3) SNR mask. The elevation mask method is one of the main methods used to remove NLOS satellites. With the aid of a fisheye image, it is easy to distinguish LOS and NLOS satellites. The RAIM with fault detection and exclusion (FDE) function is a well-known technique that is used for testing the consistency of a valid solution and excluding the outlier data. RAIM detects overdetermined pseudorange measurements from large residual errors and removes satellites from positioning. SNR is used for excluding low-strength signals due to reflection loss. Based on a previous study, the SNR decreased by more than $6 \mathrm{~dB}-\mathrm{Hz}$ when a direct line-of-sight signal is blocked by surrounding buildings and the signal is received only via reflections in low elevation angle. For SNR dependent elevation angle, the SNR threshold was typically set at 7 to $8 \mathrm{~dB}-\mathrm{Hz}$ below average SNR. When SNR of each satellite exceeds the threshold line, the satellite is then removed. Finally, the accuracy and availability derived from all techniques are compared to the SPP method to examine the improvement of positioning accuracy.

\section{METHODOLOGY}

In this section, three conventional satellite selection methods are described.

\subsection{Azimuth dependent elevation mask based on fisheye image}

Fisheye sky view provides sky obstacles density above a GNSS receiver. When a satellite's position is overlaid with the image, LOS and NLOS satellites can be identified. In this study, a fisheye image was obtained using SAMSUNG GEAR $360^{\circ}$ camera. The Azimuth dependent 
elevation mask was generated to distinguish sky and non-sky area by using the Skyplot function in open source software RTKLIB 2.4.3. When a satellite's position is located outside the mask, it is not used for positioning. The steps for making the mask are shown in Figure 1. The 1 hour observation data from static test used the satellite track with a heat map indicating the SNR for the GPS and GLONASS (L1-C/A). A fisheye camera was installed horizontally at the same location as the GNSS antenna. To express the elevation mask and a satellite's position at the same coordinates, Azimuth and projection adjustment were used. First, the image was rotated to adjust for azimuth such that north is upward and flipped. Second, the image was transformed to an equidistant projection using the lens calibration value. The next step was image binarization to separate sky area (white) and non-sky area (black). Last, the mask was created and displayed as a red line to show the threshold mask to exclude NLOS satellites.

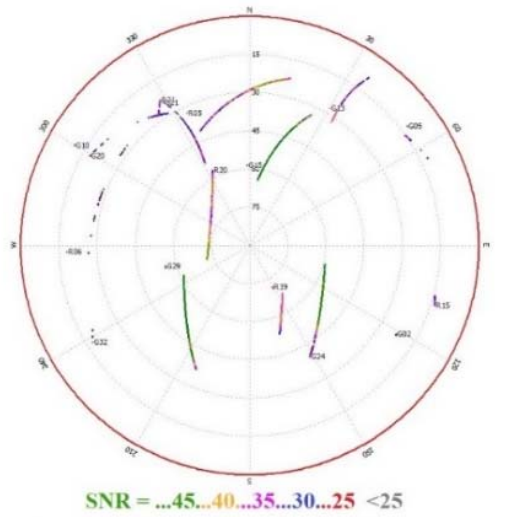

Step 1. Observation data with SNR heat map displayed using Skyplot function

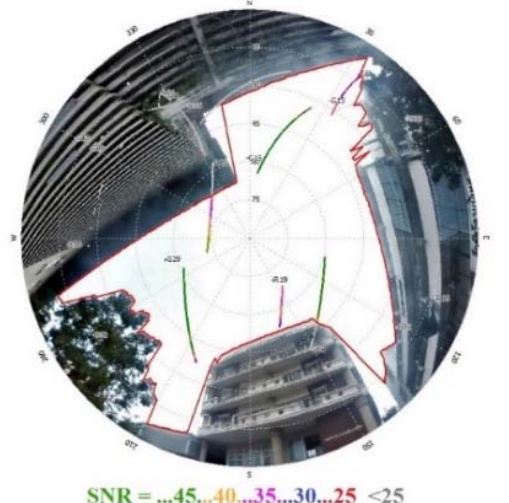

Step 3. Azimuth and projection adjustment were used to perform the image and satellite's position in same coordinate

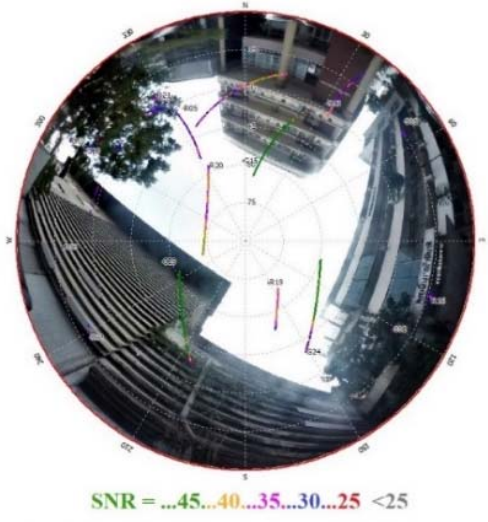

Step 2. Fisheye image was overlaid to show rough density of sky blockage

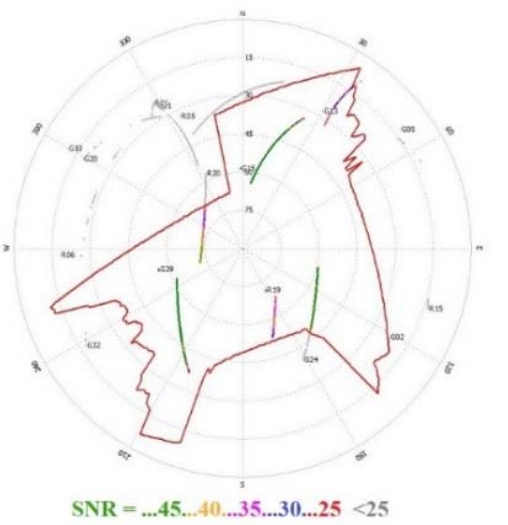

Step 4. Azimuth dependent elevation angle mask was generated to exclude NLOS satellite signal

Figure 1. Steps for generating elevation mask based on a fisheye image

\subsection{Consistency checking based on RAIM with fault detection and exclusion}

Based on the assumption, NLOS and multipath contaminated measurement produce a less consistent position solution than direct LOS measurement. The RAIM algorithm based on consistency checking is used to check integrity or the measure of trust in estimated position solutions from using pseudorange residual, which is determined as bias in ranging. The single greedy search FDE algorithm based on RAIM, which is an additional function in RTKLIB 2.4.3., was used in this study. The greedy search FDE excludes the biased satellites one by one until the test statistic satisfies the Chi-squared threshold. The process of FDE algorithm is described below: 
The linearized equation for the pseudorange measurement can be expressed as:

$$
\rho=H x+\varepsilon
$$

Where $\rho$ denotes the pseudorange measurement, $H$ denotes the observation matrix consisting of the unit LOS vector between the satellite and the estimated receiver position, $x$ is the transpose vector of the receiver states, which consists of the receiver position in three directions and receiver clock bias, and $\varepsilon$ is an error term, which is assumed to be the Gaussian noise with zero mean and covariance.

With the Least-squares estimation, the receiver states can be estimated by using equation (2):

$$
\hat{x}=\left(H^{T} W H\right)^{-1} H^{T} W \rho=K \rho
$$

The $K$ matrix is the weighted pseudo-inverse of $H$ matrix. The consistency between measurements can be determined by the pseudorange residual, as shown in equation (3):

$$
\varepsilon=\rho-H \hat{x}
$$

Next, the weighed sum of square error is calculated by equation (4). It is used as a test statistic to indicate the goodness of the least squares fit. If an outlier residual error is contained in one of the pseudorange measurements, it will cause inconsistency in the position solution.

$$
W S S E=\hat{\varepsilon}^{T} W \hat{\varepsilon}
$$

The weight matrix $W$ for the weighted least square estimation is often given as:

$$
W=\operatorname{diag}\left(\sigma_{1}^{-2}, \sigma_{2}^{-2}, \ldots, \sigma_{m}^{-2}\right)
$$

Where $\sigma_{i}$ is the standard deviation of the $\mathrm{i}^{\text {th }}$ measurement error.

The WSSE is tested by the Chi-square test based on the assumption that the pseudorange residual is a random variable, which has normal distribution. The threshold of Chi-square test is used as a constant value. It is calculated from using an appropriate probability of false alarm ( $\left.\mathrm{P}_{\mathrm{fa}}\right)$ and degree of freedom (DOF). If the test statistic is not past this threshold, one satellite will be removed and not used in positioning for the next iterative calculation. The threshold of Chi-square test can be calculated by equation (6):

$$
1-P_{f a}=\frac{1}{\tau(D O F / 2)} \int_{0}^{C T^{2}} e^{-s} s^{\frac{D O F}{2}} d s
$$

From equation (6), there is $1-P_{f a}$ of probability that the measurement cannot pass the Chisquare test. CT is the threshold of the Chi-square test, while DOF is calculated from the difference in the number of satellites and number of unknowns in the state. If the test statistic is smaller than the threshold, the solution is valid, and no satellite is removed.

To obtain an effective use in RAIM-FDE, it should be noted that fault detection and fault exclusion can be enabled when at least one DOF is required. This means that the number of satellites received must be more redundant than the unknown estimated state.

\subsection{Signal-to-noise ratio (SNR) mask}

The received signal strength can be identified by the signal-to-noise ratio (SNR) value. SNR measurements are obtained using the signal and noise power of the modulated signal at the correlator output in the receiver. Typically, SNR values are computed in $\mathrm{dB}$ scale for a specific receiver processing bandwidth, which is often unknown. However, for a more convenient way to use, receiver manufacturers always tend to record SNR values in $\mathrm{dB}-\mathrm{Hz}$ units, which relate to SNR value per $1 \mathrm{~Hz}$ bandwidth. The characteristics of SNR can be used to inspect and detect unusual behavior in a received signal. Low SNR can indicate NLOS 
reception and destructive multipath interference. Generally, SNR value should be more than $42 \mathrm{~dB}-\mathrm{Hz}$ in open-sky conditions. Under multipath conditions, SNR is typically decreased by at least 5 to $10 \mathrm{~dB}-\mathrm{Hz}$ due to signal power loss from reflection (Groves, 2013). As shown in Figure 2, when satellites GPS PRN15 and PRN29 were not blocked by building, estimated SNR value was higher than $45 \mathrm{~dB}-\mathrm{Hz}$. On the contrary, when signals were blocked and reflected by buildings (after time 06:20), SNR measurement fluctuated and dropped by more than 2 times from normal.

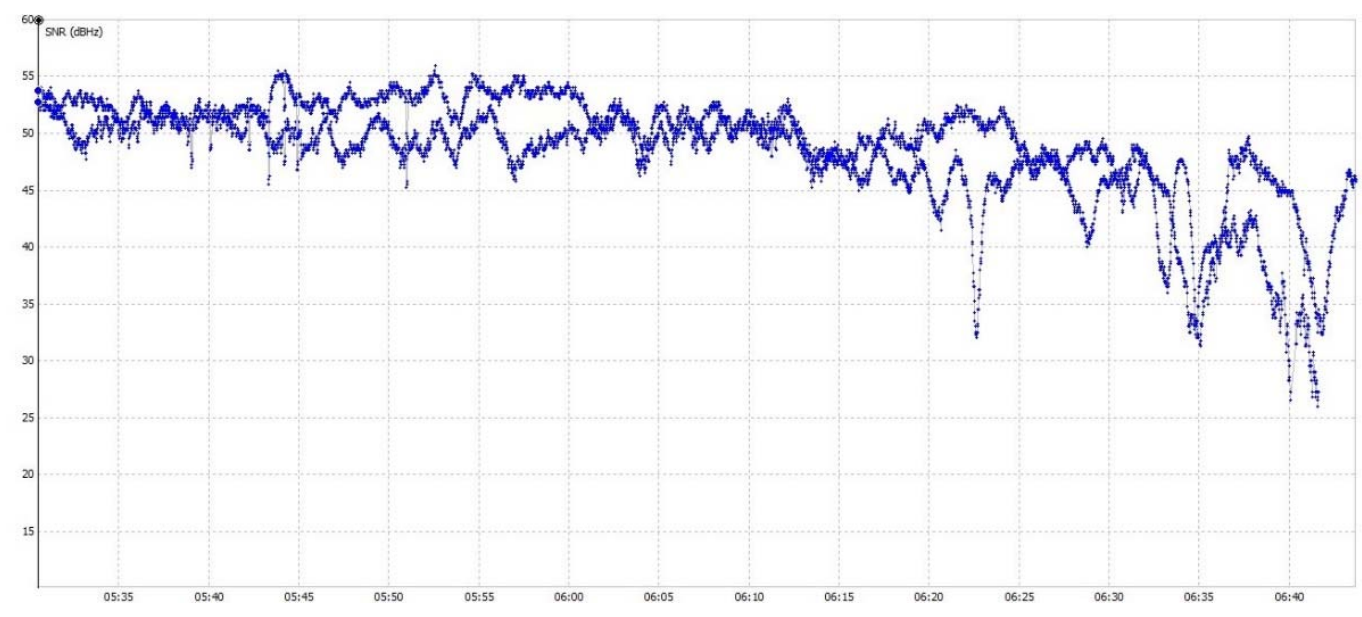

Figure 2. Example of decreased SNR value in GPS satellites

Based on this characteristic, this method is dependent on the estimated SNR measurement at the user receiver. Two types of SNR mask are performed to exclude NLOS and multipath signal. One is based on average referent SNR value, which is obtained from the multipath-free environment. Another use for SNR value depends on the satellite elevation angle. To determine the effect from multipath or NLOS satellite signal, the SNR threshold was set at approximately 7 to $8 \mathrm{~dB}-\mathrm{Hz}$ in parallel below the SNR referent line, as shown in Figure 3. When estimated SNR value exceeded the threshold, it was removed from positioning.

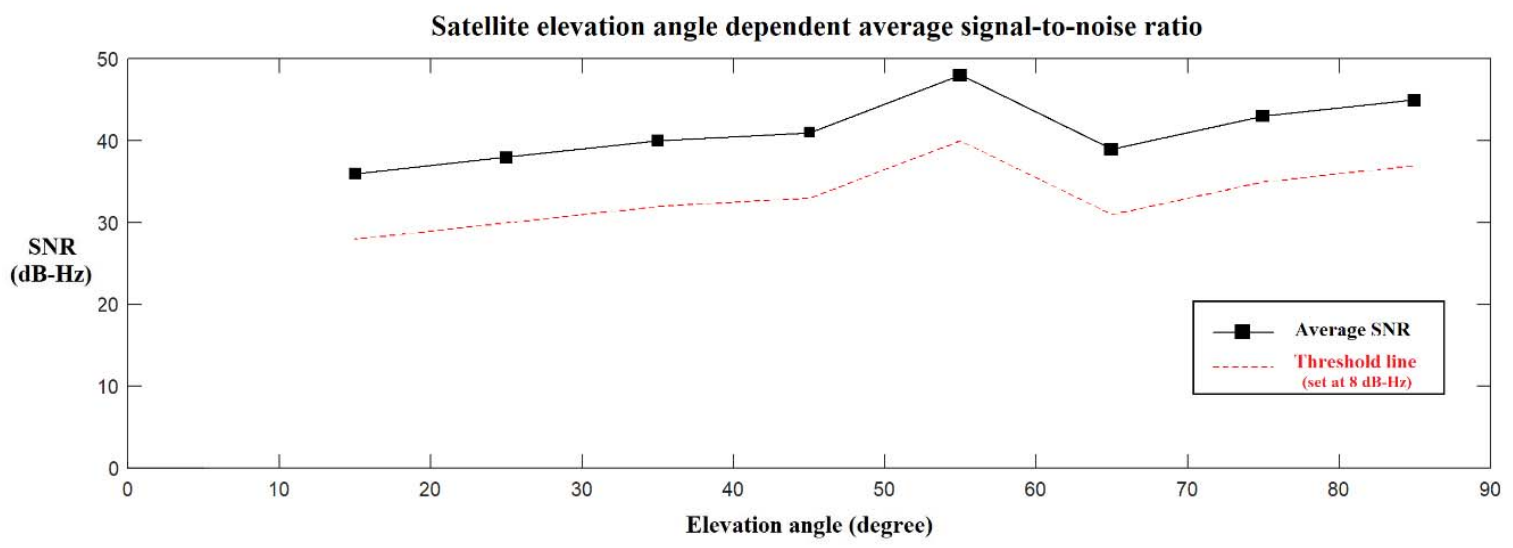

Figure 3. Elevation dependent average SNR measurement and SNR threshold

\section{EXPERIMENTAL RESULTS AND ANALYSIS}

To examine the effect of the satellite selection methods on multipath error, a preliminary 1 hour SPP in real-time $1 \mathrm{~Hz}$ test was performed in a multipath environment at Chulalongkorn University, located in Bangkok, Thailand. 


\subsection{Test data}

A 1 hour SPP test was performed in a location surrounded by mid-rise buildings. The GNSS antenna and receiver Trimble R7, which is compatible for GPS and GLONASS, were installed at the front of the Department of Survey Engineering building, Chulalongkorn University, as shown in Figure 4. GNSS data were collected at a rate of $1 \mathrm{~Hz}$ over a 1 hour period. For obtaining the sky image, a fisheye camera (SAMSUNG GEAR $360^{\circ}$ ) was installed on the top of an antenna with the north side up. The rough density of sky obstructions above GNSS antenna is shown in Figure 4.
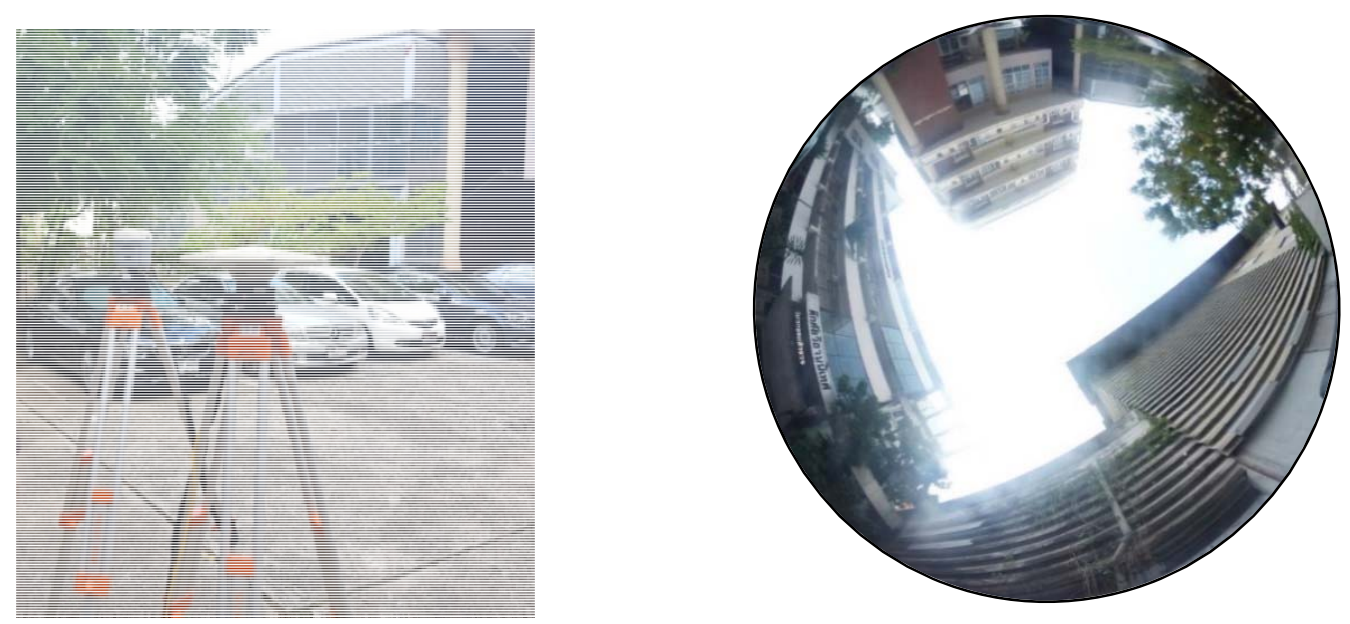

Figure 4. Testing environment and fisheye sky image

\subsection{Analysis condition}

The measurement of GPS and GLONASS on L1 frequency was used to tested by using the proposed satellite selection methods. To check the quality of the signal, observations had to pass certain basic conditions: satellite elevation angle over $15^{\circ}$ and GDOP over 10 .

To generate the elevation mask, which is used for roughly detecting obvious NLOS satellites, a fisheye image was primarily adjusted by flipping and rotating without using the parameters from Len's calibration. The mask was recorded as a text file, which expressed the elevation for each azimuth angle. To validate the margin of the mask, NLOS and LOS received satellites followed the conditions below:

$$
\begin{aligned}
\text { LOS } & =\{\text { Elevation (satellite, azimuth) }>=\text { Mask (azimuth) }-6\}: \text { Used for positioning } \\
\text { NLOS } & =\{\text { Elevation (satellite, azimuth) }<\text { Mask (azimuth) }-6\}: \text { Not used for positioning }
\end{aligned}
$$

For using RAIM-FDE to test whether or not the solution was valid and reject invalid solutions, GDOP threshold was set at 10.0 and significant value in the Chi-square distribution was set at $0.001(0.01 \%)$. After all iterations, the estimated position with the minimum squared residuals was selected as the final solution.

For SNR mask techniques, the threshold was set at $8 \mathrm{~dB}-\mathrm{Hz}$ in parallel below the SNR referent line. Referent average SNR obtained from an open-sky environment was equal to 44 $\mathrm{dB}-\mathrm{Hz}$. Then, SNR threshold was set at $36 \mathrm{~dB}-\mathrm{Hz}$ as a constant value for all elevation angles. For elevation dependent SNR mask, SNR threshold was set differently in each elevation angle, as shown in Table 1. 
Table 1. Elevation dependent SNR mask

\begin{tabular}{|c|c|c|c|c|c|c|c|c|c|}
\hline $\begin{array}{c}\text { Elevation } \\
\text { (degrees) }\end{array}$ & $<5$ & 15 & 25 & 35 & 45 & 55 & 65 & 75 & $>85$ \\
\hline $\begin{array}{c}\text { SNR threshold } \\
\text { (dB-Hz) }\end{array}$ & 0 & 28 & 30 & 32 & 33 & 40 & 31 & 35 & 37 \\
\hline
\end{tabular}

\subsection{Results}

The results of the two positioning methods were compared: SPP without additional functions of the satellite selection methods versus SPP using the proposed satellite selection methods: the elevation mask based on a fisheye image, the square residual test statistic on RAIM-FDE or the SNR masks.

According to a static data analysis by using L1 for GPS and GLONASS, the average of satellites received and GDOP was 7 and 3.8, respectively. For SNR measurement, average SNR of GPS was approximately $42 \mathrm{~dB}-\mathrm{Hz}$. Meanwhile, average SNR of GLONASS was less than GPS $2 \mathrm{~dB}-\mathrm{Hz}$ and seemed to fluctuate more at a low elevation angle, as shown in Figure 5. When using the constellation of GPS and GLONASS, the average SNR was equal to $41 \mathrm{~dB}-\mathrm{Hz}$ and had a peak (48 dB-Hz) in elevation angle at $55^{\circ}$.

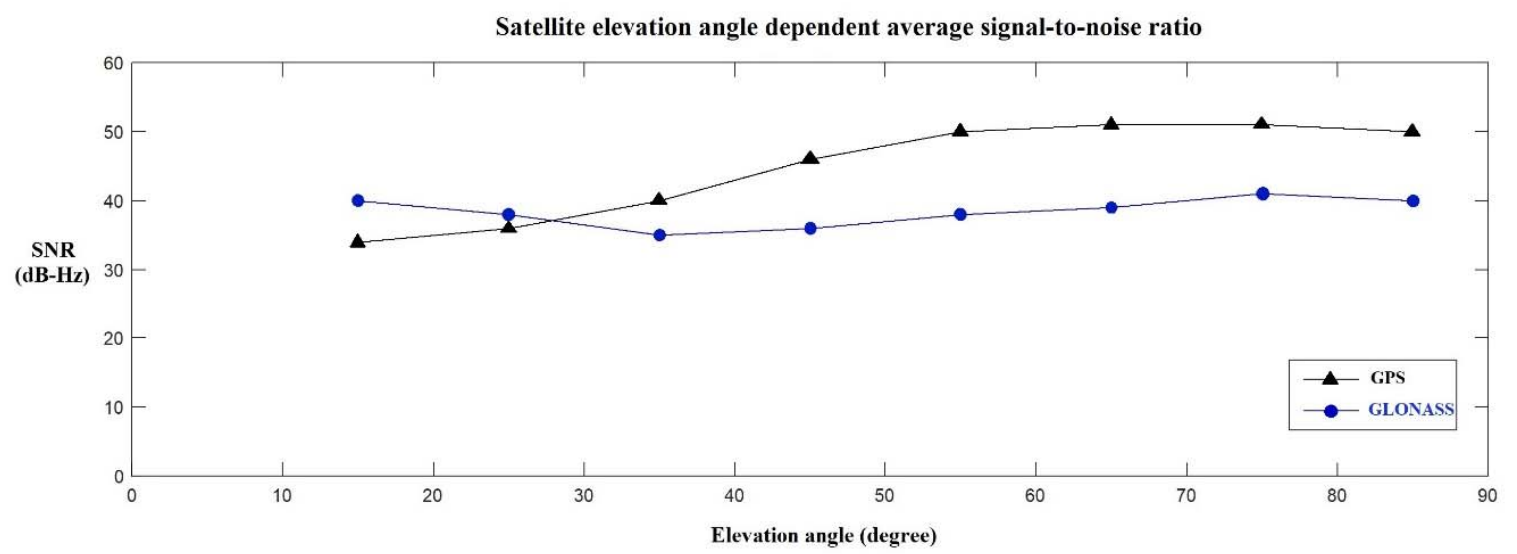

Figure 5. Average SNR of GPS and GLONASS

The magnitude of the multipath on L1 is less than 2 meters and the root mean square error (RMSE) is equal to 0.62 meters. As shown in Figure 6, multipath appeared in almost all elevations, even at high-elevation angles, but decreased to less than 1 meter in a range from $40^{\circ}$ to $50^{\circ}$. 


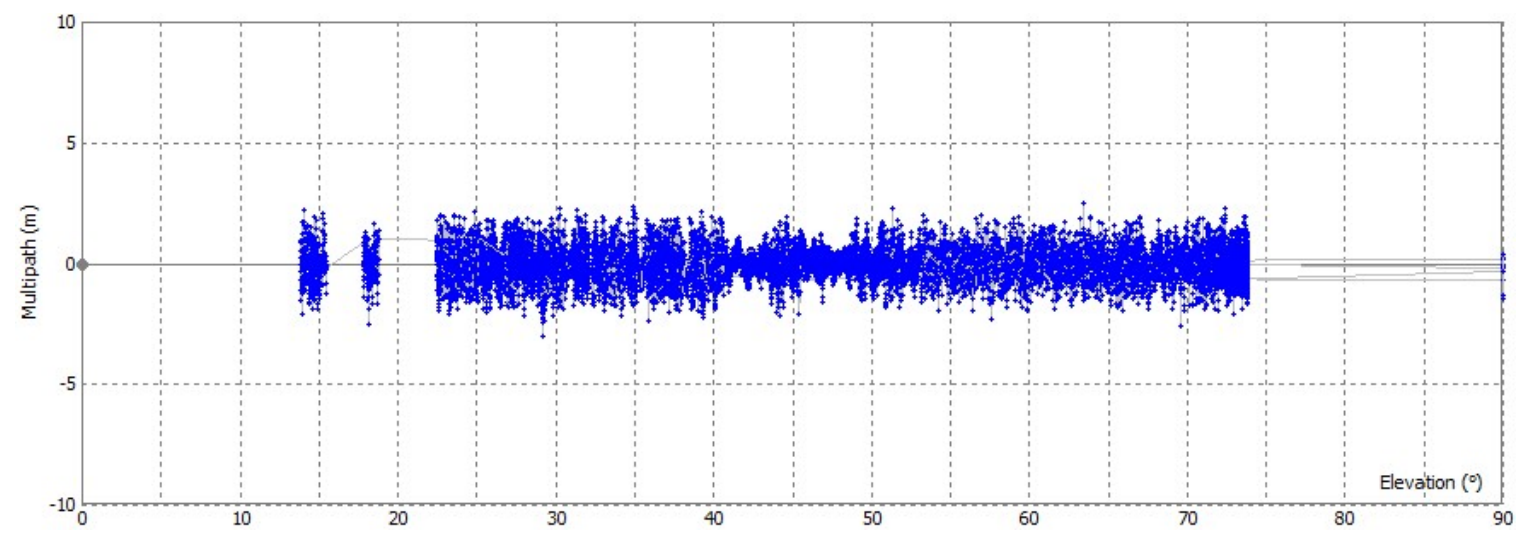

Figure 6. Magnitude of multipath on L1 using GPS and GLONASS

Before applying the satellite selection methods, a simple evaluation using GNSS constellation was performed to confirm the positioning accuracy improvement. By using a combination of GPS and GLONASS constellation compared to using GPS alone, horizontal and vertical errors were decreased by 3.22 meters and 6.54 meters, respectively. After applying the proposed satellite selection methods in SPP, the average number of valid satellites, the standard deviation (STD) for both horizontal and vertical error in the fixed solution, and satellite availability are shown in Table 2.

Table 2. Results for average number of valid satellites, positioning accuracy and availability

\begin{tabular}{|l|c|c|c|}
\hline Method & $\begin{array}{c}\text { Average of } \\
\text { valid satellites }\end{array}$ & STD (m.) & Availability \\
\hline SPP & 6.67 & $\begin{array}{c}\mathrm{H}=7.03 \\
\mathrm{~V}=14.91\end{array}$ & $\begin{array}{c}4126 / 4393 \\
=93.92 \%\end{array}$ \\
\hline $\begin{array}{l}\text { SPP } \\
\text { + RAIM FDE }\end{array}$ & 6.62 & $\mathrm{H}=10.03$ & $\begin{array}{c}4346 / 4393 \\
=98.93 \%\end{array}$ \\
\hline $\begin{array}{l}\text { SPP } \\
\text { SNR mask 36 dB-HZ }\end{array}$ & 5.54 & $\mathrm{~V}=16.56$ & $\begin{array}{c}\mathrm{H}=3.74 \\
\mathrm{~V}=12.99\end{array}$ \\
\hline $\begin{array}{l}\text { SPP } \\
\text { + SNR dependent elevation } \\
\text { mask (threshold 8 dB-HZ) }\end{array}$ & 6.12 & $\mathrm{H}=6.62$ & $4024 / 4393$ \\
& & $\mathrm{~V}=14.99$ & $=91.60 \%$ \\
\hline
\end{tabular}

Significant errors 100 meters occurred when using RAIM with a single FDE test. RMSE of the residual was equal to $3.032 \mathrm{~m}$. As a result, both horizontal and vertical positioning accuracy were reduced by $42.67 \%$ and $11.07 \%$, respectively, compared to the SPP method. Unexpectedly, the result from elevation mask could not be processed. There are only 400 epochs from 4393 epochs (9.1\%) when the number of satellites received is more than 4 . In addition, 2 to 3 LOS satellites are obviously not enough for positioning. These showed unavailability and unreliability in the solution. For the results from using SNR masks, they displayed more effectiveness. When the SNR threshold was set at $36 \mathrm{~dB}-\mathrm{Hz}$, horizontal and vertical accuracy was improved by $46.80 \%$ and $12.88 \%$, respectively. Last, when applying elevation dependent SNR mask, horizontal accuracy improved by 5.83\%, but vertical accuracy reduced by $5.37 \%$ compared to the SPP method. 


\section{CONCLUSIONS}

This study aimed to reduce the pseudorange multipath in an urban environment. To achieve this purpose, satellite selection methods based on fisheye sky imaged for improvement of SPP performance were evaluated. Four techniques were executed to find optimum position solutions: 1) RAIM-FDE test based pseudorange residual, 2) elevation angle mask 3) SNR mask, and 4) elevation dependent SNR mask. Our preliminary results showed possible impacts on the accuracy of GNSS SPP. The FDE technique and elevation mask based on fisheye image are less effective, particularly when the number of satellites is insufficient or there are two or more invalid measurements. For SNR mask technique, horizontal accuracy was improved compared to the SPP method. Specifically, SNR mask techniques set at $36 \mathrm{~dB}-\mathrm{Hz}$ for all elevations showed the best improved accuracy both horizontally and vertically. This is because multipath errors are mainly caused by NLOS reception in urban environments. The NLOS reception usually has weak SNR but enters at a relatively high elevation. As a result, SNR-based technique is more appropriate than other proposed techniques. Further research should be conducted to understand if these effects have a physical explanation model and to establish a comprehensive analysis model. The further study will focus on studying in a longer period to clarify the characteristic of multipath signal and correlation with other variables. Other environmental conditions such as closed to high rise building, between mid-rise building, and so on, and new receiver such as quadconstellation GNSS receiver will be included to future study.

\section{REFERENCES}

Dammalage T.L., Satirapod C, Kibe S. \& Ogaja C. (2010) Wavelet transform application to C/A Code multipath mitigation at GPS reference stations for improved differential GPS corrections, Survey Review, Vol. 42, 240-255.

Groves, P. D. (2013). GNSS Solutions: Multipath vs. NLOS signals. How Does Non-Lineof-Sight Reception Differ From Multipath Interference, Inside GNSS, Vol.8, 40-44.

Groves, P.D., Height, Z. J., Rudi M. \& Strode P. (2013). A Portfolio Approach to NLOS and Multipath Mitigation in Dense Urban Area. Proc. of the 26th International Technical Meeting of the Satellite Division of the Institute of Navigation 2013, Nashville, U.S.A, 3231-3247.

Hsu, L.T., Jan, S.S., Groves, P. \& Kubo, N. (2016) Multiple Faulty GNSS Measurement Exclusion based on Consistency Check in Urban Canyons, IEEE Sensors Journal, Vol. 17, 1909-1917. doi:10.1109/JSEN.2017.2654359

Iwase, T., Susuki, N. \& Watanabe, Y. (2013) Estimation and Exclusion of Multipath Range Error for Robust Positioning, GPS Solutions, Vol. 17, 53-62. doi:10.1007/s10291-012-0260-1

Lin, K.Q., Deng, Z.L. \& Yin, L. (2018) Effective Multipath Mitigation Methods for RTK in Urban Environments. Proc. of China Satellite Navigation Conference (CSNC) 2018, Harbin, China, 565-576.

Matera E.R., Peña A.J.G., Julien, O., Milner, C. \& Ekambi, B. (2019) Characterization of Line-of-Sight and Non-Line-of-Sight Pseudorange Multipath Errors in Urban Environment for GPS and Galileo, Proc. of the 2019 International Technical Meeting of The Institute of Navigation, Reston, Virginia, U.S.A., January, 177-196.

Sánchez, J.S., Gerhmann, A., Thevanon, P. \& Brocard, P. (2017) Use of a Fisheye Camera for GNSS NLOS Exclusion and Characterization in Urban Environments, Proc. of the 2016 International Technical Meeting of The Institute of Navigation, Monterey, California, U.S.A., January, 283-292. 
Satirapod C. \& Rizos C. (2005) Multipath Mitigation by Wavelet Analysis for GPS Base Station Applications, Survey Review, Vol. 38, 2-10.

Špánik P. \& Hefty J. (2017) Multipath Detection with the Combination of SNR Measurements - Example from Urban Environment, GEODESY AND CARTOGRAPHY, Vol. 66, 305-315.

Suzuki, T. (2011) High - Accuracy GPS and GLONASS Positioning by Multipath Mitigation Using Omnidirectional Infrared Camera, IEEE International Conference on Robotic and Automation, 311-316. doi:10.1109/ICRA.2011.5980424

Suzuki, T. \& Kubo, N. (2015) Simulation of GNSS Satellite Availability in Urban Environments Using Google Earth, Proc. of the ION 2015 Pacific PNT Meeting, Honolulu, Hawaii, U.S.A., April, 1069-1079.

Takasu T. (2013) GNSS RTKLIB software Version 2.4.2 Manual. Retrieved from http://www.rtklib.com/prog/manual_2.4.2.pdf. Teunissen P. J.G. \& Montenbruck O. Eds (2017). Springer Handbook of Global Navigation Satellite Systems: Switzerland. doi: 10.1007/978-3-319-42928-1.

Tokura, H. \& Kubo, N. (2014) Using Multiple GNSS Constellations with Strict Quality Constraints for More Accurate Positioning in Urban Environments, Scientific Research , Vol. 5, 85-96. doi:10.4236/pos.2014.54011

Tokura H. \& Kubo N. (2017) Efficient Satellite Selection Method for Instantaneous RTKGNSS in Challenging Environments. Trans. Japan Soc. Aero. Space Sci., Vol. 60, 221-229. doi:10.2322/tjsass.60.221

Tongleamnak, S. \& Nagakai, M. (2017) Simulation of GNSS Availability in Urban Environments Using a Paranomic Image Dataset. International Journal of Navigation and Observation, Vol. 2017. doi:10.1155/2017/8047158

Townsend B.R. \& Fenton P.C. (1994) A Practical Approach to the Reduction of Pseudorange Multipath Errors in an L1 GPS Receiver. Proc. of the 7th International Technical Meeting of the Satellite Division of The Institute of Navigation 1994, Salt Lake City, UT, U.S.A., September, 143-148.

Wang, E., Cai, M., \& Pang, T. (2012) A Simple and Effective GPS Receiver Autonomous Integrity Monitoring and Fault Isolation Approach. IEEE, 657-660. doi:10.1109/ICCECT.2012.145

Received: 2020-03-02,

Reviewed: 2020-04-22 (by J.B. Zieliński) and 2020-04-22 (by B. Oszczak),

Accepted: 2020-04-24. 